\title{
GDM progenies exhibit sex disparity in metabolism after respective therapies of insulin, glibenclamide and metformin in dams during pregnancy
}

Yao Lu

Anhui Medical University

Zhengxuan Jjang

Anhui Medical University

Yajing Jia

Anhui Medical University

Juan Liu

Anhui Medical University

Yuxin $\mathrm{Xu}$

Anhui Medical University

Qingsi Wu

Anhui Medical University

Keyang Chen ( $\nabla$ chenkeyang@ahmu.edu.cn )

Anhui Medical University https://orcid.org/0000-0003-3019-577X

Original investigation

Keywords: GDM, Offspring, Glibenclamide, Metformin, Glucose and lipid metabolism

Posted Date: September 29th, 2020

DOI: https://doi.org/10.21203/rs.3.rs-78696/v1

License: (c) (1) This work is licensed under a Creative Commons Attribution 4.0 International License.

Read Full License 


\section{Abstract}

Background $\mathbb{T}$ The drug treatments for the pregnant women with gestational diabetes mellitus (GDM) have been concerned with their effects on both mothers and offspring. The aim of this study was to compare the intergenerational effects of insulin, glibenclamide and metformin on glucose and lipid metabolism in the offspring born to GDM mice.

Methods: The murine GDM was induced by high fat diet. The offspring were grouped based on the treatments in maternal mice. ITT and GTT were performed at $4^{\text {th }}$ week and $8^{\text {th }}$ week of age, respectively. Serum levels of TC, TG, HDL-C and LDL-C plus hepatic levels of TG and TC, were respectively determined by enzymatic kits. Western blotting was conducted to detect related proteins in the livers from offspring.

Results: The normalization of the dyslipidemia, hepatic lipid abnormality and insulin insensitivity elicited by the respective therapies of insulin, glibenclamide and metformin during maternal pregnancy still persisted in the male adult offspring. Specifically, the decreases in plasma TC, TG, LDL-C levels $(29 \%$,

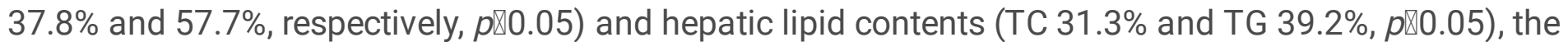
increases in hepatic phosphorylation levels of AKT, CPT1A ,PPAR- $a$ and PPAR-y $(57.1 \%, 91.7 \%, 68 \%$ and $173.3 \%$, respectively, $p \otimes 0.05)$ and the inhibition of G6Pase, PEPCK, HMGCS1 $(35.7 \%, 68.8 \%$ and $77.3 \%$ respectively, $p \varangle 0.05$ ) were still observed in the male offspring born to treated GDM mice from $4^{\text {th }}$ to $8^{\text {th }}$ week of age. Unexpectedly, the female progeny born to untreated GDM mice showed an autonomous recovery in glucose and lipid metabolism.

Conclusions: Respective treatments in GDM mice during pregnancy with insulin, glibenclamide and metformin have the long-term persisted effects in male offspring, while female progenies born to untreated dams showed an autonomous inhibition of intergenerational relay of glucose and lipid dysregulation. Our current findings may shed a new light into GDM clinical practice.

\section{Background}

GDM is defined as the onset of glucose intolerance during pregnancy ${ }^{1}$. Because uncontrolled hyperglycemia during pregnancy affects fetal development and neonatal adaptation. Adequate treatment has a direct impact on maternal and perinatal outcomes ${ }^{2}$. The fetal exposure to maternal diabetes in utero increases the risk of obesity, glucose intolerance and type 2 diabetes (T2DM) for offspring in later life ${ }^{3,4}$. For $15-60 \%$ of women with GDM, routine care such as dietary measures, physical activity, and glucose monitoring is not adequate to achieve glucose control ${ }^{5}$. Therefore, the medical therapies with hypoglycemic agents during pregnancy may be very crucial for GDM women and their offspring to maintain health. Although insulin has been commonly used in the management of GDM ${ }^{6}$, it has many disadvantages including a complicated dosing schedule, an unfavorable route of administration and reduced patient adherence. Oral hypoglycemic agents are noninvasive and easy to administer ${ }^{7}$. Recently, International Federation of Gynecology and Obstetrics (FIGO) and the UK National Institute for Health and Care Excellence (NICE), suggested insulin, glibenclamide, and metformin as appropriate first-line 
therapies for GDM ${ }^{8,9}$. The rationale for the use of glibenclamide during pregnancy is based on the similarities of the pathophysiology in GDM and T2DM. Sulfonylurea (SU) drugs have been used to treat T2DM for many decades. Metformin was reintroduced more recently as treatment for GDM. Based on the available data regarding the short-term effects, glibenclamide and metformin appear to be safe and effective for the treatment of GDM ${ }^{10,11}$. But previous studies have all stressed the lack of intergenerational effect data on offspring. The unanswered questions are mainly related to offspring's late development, growth and glucose-lipid metabolism. Therefore, the intergenerational effects of glibenclamide and metformin on the metabolic regulation in GDM offspring are currently unknown and constitute an important barrier for their use. In the present study, we used murine GDM model to explore the intergenerational impacts of respective interventions by insulin, glibenclamide and metformin during pregnancy on the offspring born to GDM mice.

\section{Material And Methods}

\section{Mice handlings}

C57BL/6J mice (8 weeks old; male mice: $20 \pm 24 \mathrm{~g}$; female mice: $17 \pm 20 \mathrm{~g}$ ) were obtained from Beijing Vital River. Mice were kept under observation for one week to acclimatize the animals to the new conditions. Mice were maintained under a $12 \mathrm{~h} \mathrm{light-dark}$ cycle at constant temperature $(23 \pm 2)^{\circ} \mathrm{C}$ and relative humidity ( $50 \pm 5$ ) \% with food (Beijing Huafukang Bioscience Co, LTD, Beijing 11027182) and water available ad libitum. The GDM model was established by being fed with high-fat diet (HFD; $60 \%$ $\mathrm{kcal}$ from fat) for $4 \mathrm{wk}$ before pregnancy ${ }^{12,13}$. The pregnant mice were obtained by proesterous normal females being left for one night to copulate with the normal males (2:1). Onset of pregnancy was determined by the presence of a copulation plug, which was defined as gestational day zero (GD0). The total successful mating for GDM group were 62, while the successful mating for normal controls were 11 (N0). On the GD6 and GD12, the dams in GDM group received a streptozotocin (STZ) injection (100 mg/kg i.p.), respectively, control dams received an equal volume of citrate buffer. The diabetic state for GDM group was confirmed when the fasting plasma glucose concentration exceeded $14 \mathrm{mmol} / \mathrm{l}$ $(252 \mathrm{mg} / \mathrm{dl}){ }^{14,15}$. The successful GDM models were randomly distributed into 4 groups (10 animals/group): GDM control without treatment (G0), GDM treated with insulin (GI0), GDM treated with glibenclamide (GG0) and GDM treated with metformin (GM0). The drug administration was conducted since the hyperglycemia was confirmed during pregnancy.

The offspring's groups were named based on their mothers' groups, respectively (8-10 pups/group), recorded as N, G, GI, GG, GM. After delivery, the fetal mice were obtained, and their fetal weights, crownrump lengths were measured. The offspring were fostered until they were weaned after 3 weeks of age. All of offspring in experimental groups were weighed on days of $3,7,10,14,17$ and 21, respectively, during lactation. Body weights and plasma glucose were measured once a week after weaning. ITT and GTT experiments were performed respectively at 4th week and 8th week of age.

\section{Insulin Treatment}


The therapeutic dose to the GI0 group was 7 to $10 \mathrm{U}$ (human long-acting insulin, Novo Nordisk A/S, Denmark) for the initial subcutaneous injection ${ }^{16}$. Subsequent daily injections were adjusted according to the plasma glucose level, with $1 \mathrm{U}$ each time.

\section{Metformin And Glibenclamide Therapy}

The mice were administered by gavage with $20 \mathrm{mg} / \mathrm{kg} / \mathrm{d}$ of glibenclamide for GG0 group ${ }^{17}$, and by gavage with $300 \mathrm{mg} / \mathrm{kg} / \mathrm{d}$ of metformin for GM0 group. The volume of gavage administration was $1 \mathrm{ml} / 100 \mathrm{~g}$ body weight.

\section{Glucose Tolerance Test (GTT) And Insulin Tolerance Test (ITT)}

GTT were performed on murine offspring after $8 \mathrm{~h}$ fasting by injecting the glucose ( $2 \mathrm{mg} / \mathrm{g}$ body weight) into the intraperitoneal cavity and plasma glucose was measured via tail blood immediately before and after $0.5,1,1.5,2$ and $2.5 \mathrm{~h}$ injections using an Accu-Check glucometer (Roche, Germany). ITT were performed on $4 \mathrm{~h}$ fasted conscious offspring respectively by injecting human insulin ( 4 weeks, $0.45 \mathrm{mU} / \mathrm{g}$ ; 8 weeks $₫ 0.65 \mathrm{mU} / \mathrm{g}$ body weight; SIGMA, USA) into the intraperitoneal cavity and plasma glucose was measured via tail blood immediately before and after 20, 40, 60, 80, 100 and 120 min injections using an Accu-Check glucometer (Roche, Germany).

\section{Tissue Collection And Biochemical Assay}

After 8 weeks, blood samples were respectively collected from the orbital venous plexus in offspring after $10 \mathrm{~h}$ fasting when the mice were anesthetized with ketamine $(100 \mathrm{mg} / \mathrm{kg})$, acepromazine $(10 \mathrm{mg} / \mathrm{kg})$, and xylazine $(100 \mathrm{mg} / \mathrm{kg})$. Whole blood obtained from offspring was centrifuged at $3000 \mathrm{~g}$ at $4{ }^{\circ} \mathrm{C}$ for 20 min to collect plasma. The livers were immediately removed and rinsed with cold phosphate-buffered saline (PBS) and stored at-80 ${ }^{\circ} \mathrm{C}$ after dip-frezzing in liquid nitrogen. Insulin was analyzed using an enzyme-linked ELISA kit (Elabscience, Wuhan, China). Plasma levels of TC, TG, HDL-C, LDL-C, and hepatic levels of TG and TC, were determined respectively using enzymatic kits (Nanjing Jiancheng Bioengineering Institute, Jiangsu, China).

\section{Western blotting}

Liver homogenates were prepared with RIPA lysis buffer containing a complete protease inhibitor. Lysates were centrifuged at $12000 \mathrm{~g}, 4^{\circ} \mathrm{C}$ for $15 \mathrm{~min}$. The supernatant extracts were quantified for protein concentration assay by BCA. 30-50 $\mu \mathrm{g}$ of denatured proteins were loaded, resolved by $10-15 \%$ SDSPAGE and transferred to PVDF membranes, that were incubated in 5\% non-fat milk at room temperature for $1 \mathrm{~h}$, and then incubated with the appropriate primary antibodies respectively, overnight at $4{ }^{\circ} \mathrm{C}$. Membranes were then washed and incubated with secondary antibodies at room temperature for 1-2 $\mathrm{h}$. Target proteins were detected by enhanced chemiluminescence $(E C L)$.

\section{Statistical Analysis}


Data are expressed as mean \pm SEM. The physiology and biochemistry parameters in dams were analyzed using one-way ANOVA followed by the SNK comparisons test. A value of $P<0.05$ was considered to be statistically significant. ITT, GTT were additionally analyzed using glucose area under the curve (AUC), calculated using trapezoidal integration.

\section{Results}

\section{Respective interventions of insulin, glibenclamide and metformin during pregnancy improved the hyperglycemia and insulin resistance in GDM dams.}

The whole experimental scheme is shown in Fig. 1A. Based on current data, the growth rate of dams in the untreated diabetic G0 group was significantly higher than those in the drug-treated groups (Fig. 1B), indicating that the respective therapies of insulin, glibenclamide and metformin just exerted substantial reduction effects on the maternal body weights during pregnancy (Fig. 1B). In addition, the fasting plasma glucose levels in untreated GO group were dramatically higher than those in NO group, and the interventions of three drugs respectively and dramatically ameliorated this hyperglycemia (Fig. 1C).

As expected, the GDM dams showed the significant hyperinsulinemia, which could be normalized by the respective three drug therapies during pregnancy $(P<0.05$, Fig. 1D). Similarly, untreated GDM dams showed significantly elevated HOMA-IR, which could be drastically alleviated by the respective drug therapies $(P<0.001$, Fig. 1E).

\section{Respective interventions of three drugs during maternal pregnancy normalized fetal body weights and improved offspring overweight during lactation}

After G18, the dams delivered the fetus which were collected and measured respectively. The crown-rump length and body weights of the fetus born to untreated diabetic $G$ group were significantly higher than those in $\mathrm{N}$ group (Fig. 2A and B), but the therapies of insulin, glibenclamide and metform in dams respectively normalized these characteristics. Through the lactation (3-21days), the growth rates of offspring born to $\mathrm{G}$ group were sharply faster than those in $\mathrm{N}$ group $(P<0.05$, Fig. $2 \mathrm{C})$, suggesting that the overweight in fetus elicited by untreated GDM in dams exerted the intergenerational impact on their offspring, while the respective interventions of three drugs significantly and similarly ameliorated the effects $(P<0.05$, Fig. 2C).

\section{Respective interventions of three drugs during maternal pregnancy normalized the fasting plasma glucose and growth rate in male offspring at adulthood}

After three weeks of lactation, male and female offspring were kept in separate cages. Body weights and plasma glucose were measured once a week. The growth rates of female offspring in N, GI, GG and GM groups were gradually reached the same levels including those in the untreated G group (Fig. 3A). Likewise, the fasting plasma glucose levels in female offspring showed the same convergent trend from 4 to 8 weeks of age. These results suggested a gradually autonomous correction of overgrowth and 
hyperglycemia in female progenies (Fig. 3A and B). However, the male offspring in the untreated $\mathrm{G}$ group kept growing faster than those in $\mathrm{N}, \mathrm{Gl}, \mathrm{GG}$ and $\mathrm{GM}$ groups, and respective interventions during maternal pregnancy showed significant intergenerational improvements on the body weights in male offspring at adulthood (Fig. 3C). Similarly, the male progenies in untreated $\mathrm{G}$ group demonstrated the persisted rising hyperglycemia compared to the $\mathrm{N}$ group, which could be normalized by the three respective therapies during maternal pregnancy (Fig. 3D).

\section{Respective therapies of three drugs in dams normalized the glucose tolerance and insulin sensitivity in all male offspring at adulthood}

In order to examine the offspring's insulin sensitivity after weaning and when they reach adulthood, we carried out an acute insulin challenge test in the female and male offspring, respectively. As shown in Fig. 4, After exogenous insulin injection in offspring with 4 weeks of age, the plasma glucose levels in the female offspring born to the untreated GDM mice stayed slightly higher than those in N, GI, GG and GM groups (Fig. 4A), while the same tests in male progeny showed significant elevation in untreated group (Fig. 4B). The results suggest that insulin resistance still exist in the male offspring with 4 weeks of age which could be totally corrected by the three respective drug therapies, and that female offspring may exhibit an autonomous alleviation trend. For the same test in the offspring with 8 weeks of age, after the insulin injection, the blood glucose levels in the female offspring born to the untreated $\mathrm{G}$ group was not different from those in intervention groups and normal control group (Fig. 4C), indicating an automatic recovery of insulin sensitivity in female adult offspring. While the same test showed that the blood glucose levels in the male offspring born to the untreated dams in $\mathrm{G}$ group were still markedly higher than those in $\mathrm{N}, \mathrm{Gl}, \mathrm{GG}$ and GM groups, suggesting that the intergenerational intervention effects were still persisting for male adult offspring (Fig. 4D). To explore the offspring's ability to acutely handle the glucose loading, GTT experiments were performed in mice at 4 and 8 weeks of age, respectively. After exogenous glucose injection into female offspring with 4 weeks of age, the peak of glucose levels in the untreated group appeared at 30 minutes that was slightly different from the 30-minute-peaks in $\mathrm{N}$ group and other treated groups (Fig. 4E). On the contrary, the GTT tests in male progenies at 4 weeks old demonstrated substantially higher blood glucose levels in untreated group than $\mathrm{N}$ and other treated groups (Fig. 4F). These results suggest that impaired glucose tolerance induced by untreated GDM in dams still exists in the male offspring with 4 weeks of age. Furthermore, the GTT tests conducted in female offspring with 8 weeks of age showed no any significant difference for postprandial glucose levels in all 5 groups (Fig. 4G). Whereas, the same test performed in the male offspring with 8 weeks old revealed that the postprandial glucose levels in GI, GG and GM groups were significantly lower than those in the untreated $\mathrm{G}$ group, suggesting that three interventions in maternal mice still elicited the amelioration effects for the male offspring even when they reach adulthood (Fig. 4H).

The normalization of hyperlipidemia and hepatic lipid contents by respective drug therapies in dams during pregnancy still persisted in male offspring at adulthood 
The lipid profiles in each group are shown in Fig. 5. For the female offspring with 8 weeks of age, there were no significant differences in serum TG, TC, LDL-C, and HDL-C levels in all groups. These results indicate the possible autonomous correction of dyslipidemia in adult female offspring $(P>0.05$, Fig. 5A$D$ ). For the male progenies, compared with $\mathrm{N}$ group, serum TG, TC, and LDL-C levels were significantly elevated in those born to the untreated GDM dams $(P<0.05$, Fig. 5E-G), while there was no difference in HDL-C levels in each group ( $P>0.05$, Fig. $5 \mathrm{H})$. For the female offspring with 8 weeks of age, there were no significant differences in liver TG, TC levels in all groups, including those born to the untreated GDM maternal mice ( $P>0.05$, Fig. $5 \mathrm{I}$ and $\mathrm{J})$. For the male progenies, compared with $\mathrm{N}$ group, liver TG, TC levels were significantly elevated in the untreated $\mathrm{G}$ group $(P<0.05$, Fig. $5 \mathrm{~K}$ and $\mathrm{L})$.

\section{The female adult offspring demonstrated the autonomous normalization of insulin sensitivity, glucose and lipid metabolism}

To evaluate insulin resistance in offspring, we measured fasting plasma glucose and serum insulin in male and female progenies with 8 weeks of age. The results showed that there were no differences in fasting plasma glucose and serum insulin levels between each group for all female offspring $(P>0.05$, Fig.S1A and B). To explore the mechanism by which the interventions impact insulin sensitivity, we tested the hepatic levels of p-AKT (Thr473, Ser308) and p-FOX01. Our results showed that the levels of p-AKT and p-FOXO1 in female adult progenies with 8 weeks of age did not differ between all groups, including the untreated G control group (Fig. 6A, and B). PEPCK and G6Pase catalyze committed steps of gluconeogenesis, thus play important roles in glucose homeostasis. The protein expressions of PEPCK and G6Pase in female adult progenies did not differ between all groups either (Fig. 6C). SREBP2 and HMGCS1 play an important role in the regulation of cholesterol synthesis. Our immunoblots showed that the protein expressions of hepatic SREBP2 and HMGCS1 in female adult progenies did not differ between the treated and untreated groups (Fig. 6D). ACL plays a fundamental role in lipogenesis and steroidogenesis. Its activation provides the building blocks for fatty acid biosynthesis. The ratios of $p$ ACL to ACL did not differ between all groups (Fig. 6E). CPT1A is a rate-limiting enzyme for fatty acid $\beta$ oxidation. It can transfer medium- and long-chain fatty acids into mitochondria for $\beta$-oxidation, which reduces the deposition of lipids in peripheral tissues. The hepatic protein expressions of CPT1A in female adult progenies did not differ between the untreated and treated groups (Fig. 6F). PPAR- $\alpha$ and PPAR- $\gamma$ are the nuclear transcription factors. They are not only key regulators of adipose tissue differentiation and lipid metabolism, but also necessary molecules to maintain insulin sensitivity. The hepatic protein expressions of PPAR- $a$ and PPAR- $y$ in female adult progenies did not differ between the untreated and treated groups (Fig. 6G).

\section{The normalization of insulin sensitivity, glucose and lipid metabolism by respective therapies of three drugs in dams persisted in male offspring at adulthood}

The HOMA-IR in female adult offspring from the untreated $\mathrm{G}$ group was not different from those in other invention groups ( $P>0.05$, Fig.SC). However, the male adult offspring born to the untreated GDM maternal mice showed significantly elevated HOMA-IR owing to the substantially enhanced FPG levels in 
the $\mathrm{G}$ group, which could be normalized by the respective therapies of insulin, glibenclamide and metformin during maternal pregnancy $(P<0.05$, Fig.SD and SF), despite the similar plasma insulin levels between all groups for male adult offspring ( $P>0.05$, Fig.SE). Likewise, to explore the mechanism by which the interventions enhanced insulin sensitivity, we found that the hepatic p-AKT (Thr473, Ser308) and p-FOXO1 ratios in male adult progenies born to the untreated GDM dams were still significantly reduced compared with the $\mathrm{N}$ group. However, the treatments restored $\mathrm{p}$-AKT and p-FOXO1 levels in male adult offspring with 8 weeks of age, similar to those in $\mathrm{N}$ group (Fig. 7A and B). The significant increases in PEPCK and G6Pase were observed in livers of male adult offspring in the untreated $\mathrm{G}$ group, compared to those in $\mathrm{N}$ group, which were normalized by the three drug therapies during maternal pregnancy (Fig. 7C), except that metformin has no inhibitory effect on the expression of G6Pase in the livers of male offspring (Fig. 7C). SREBP2 in male adult progenies did not differ between all groups (Fig. 7D). HMGCS1 was significantly increased in livers of male adult offspring in untreated $\mathrm{G}$ group, compared to $\mathrm{N}$ group, and the elevations were sharply inhibited by three drugs respectively (Fig. 7D). The ratios of p-ACL to ACL were significantly decreased in the untreated G group compared to the $\mathrm{N}$ group (Fig. 7E), suggesting the increased ACL activity in $\mathrm{G}$ group. However, the activity could be dramatically inhibited by the three drug interventions compared to $\mathrm{N}$ group (Fig. 7E). The protein expressions of PPAR- $\mathrm{a}$ and CPT1A were significantly decreased in livers of male adult offspring in untreated $\mathrm{G}$ group, compared to those in $\mathrm{N}$ group (Fig. 7F and G). Nevertheless, the PPAR- $a$ and CPT1A levels could be normalized in male adult progenies born to the treated dams, indicating an intergenerational effect by the three drug interventions during maternal pregnancy (Fig. 7F and G). Likewise, the protein expressions of PPAR-y significantly decreased in the untreated $\mathrm{G}$ group compared to the $\mathrm{N}$ group (Fig. 7G), which however could be significantly enhanced by the three drug therapies in dams.

\section{Discussion}

The children born to the mothers with GDM are prone to develop chronic diseases such as obesity, impaired glucose tolerance, and T2DM in adulthood ${ }^{17}$. In the present study, our HFD + STZ-induced GDM mouse model was just established by the dramatic elevation of blood glucose concentration. More importantly, our data clearly showed the significant hyperinsulinemia and insulin resistance in GDM mouse models that mimics the human GDM state (Fig. 1D and 1E). In particular, the hyperglycemia in our GDM mice could be significantly ameliorated by the glibenclamide, a representative of insulin secretion enhancer (Fig. 1C).

Our current results show that insulin, glibenclamide and metformin respectively alleviated fetal overgrowth and improved offspring's body weights during breastfeeding. These drugs possibly worked by improving intrauterine hyperglycemia, inflammation and oxidation, etc.

GTT is widely applied to assess glucose homeostasis. Glucose stimulates the pancreas to secrete insulin, which increases the utilization of blood glucose. A reduction in glucose homeostasis in diabetic mice may result in marked hyperglycemia by the GTT ${ }^{18,19}$. Correspondingly, ITT is widely applied to assess insulin sensitivity. 
In the present study, we unexpectedly found that the female offspring born to the untreated GDM dams automatically became normalized when reach their adulthood with 8 weeks of age, although the normalization trend occurred since they were at 4 weeks old. These findings may be similar with the reports from Jun Ren et al. and Di Xiao et al, suggesting that female offspring might have better glucose tolerance and insulin sensitivity than male counterparts 17,20 . The rational explanation of the gender differences might be attributed to the sexual hormones.

The prevalence of T2DM has shown gender disparities. Premenopausal women exhibit enhanced insulin sensitivity and reduced incidence of T2DM compared with age-matched men, but this advantage disappears after menopause with disrupted glucose homeostasis, in part owing to a reduction in circulating $17 \beta$-estradiol (E2) ${ }^{21}$. Both clinical and animal studies show a strong correlation between estrogen deficiency and metabolic dysfunction 22,23 . The reduction of estrogen in postmenopausal women accelerates the development of insulin resistance and T2DM ${ }^{24}$. Clinical trials of estrogen replacement therapy in postmenopausal women demonstrated an amelioration of insulin resistance and reductions of plasma glucose level and incidence of T2DM ${ }^{25}, 26$. Hui Yan et al. demonstrated that E2 improves insulin sensitivity and suppresses hepatic gluconeogenesis through inhibition of Foxo1 via activation of ERa-PI3K-Akt signaling by investigating the action of E2 on glucose homeostasis in male and ovariectomized female control and liver-specific Foxo1 knockout mice ${ }^{27}$. It was reported that estrogen receptor a (ERa) regulated $\beta$-cell formation during pancreatic development ${ }^{28}$. However, some studies also indicated that the serum testosterone level of male patients and male animals with obesity and related diseases was significantly lower than controls, which was closely related to the disorder of glucose and lipid metabolism 29, 30 . Exogenous testosterone supplementation could alleviate the occurrence and development of obesity and related diseases and improve glucose and lipid metabolism, which confirmed the important role of androgen in the occurrence of these diseases and the regulation of glucose and lipid metabolism ${ }^{31}, 32$. However, unlike men, women with too high androgen levels, such as patients with polycystic ovary syndrome, may develop obesity, disorders such as glucose and lipid dysregulation, insulin resistance, and diabetes $29,30,33$. Therefore, the better compensation of the glucose metabolic function in female offspring might be due to the relative high concentration of estrogen in female pups. Di Xiao et al. also reported that FGR female offspring have higher mRNA expression of insulin receptor in livers than the male progenies ${ }^{34}$. Thus the improved hepatic insulin/IGF1 signaling pathways may be one of the reasons for the normalized insulin sensitivity. In addition, several studies indicated long-term exercise training improves insulin sensitivity in both liver and muscle ${ }^{35}$. Female mice naturally exhibited a higher physical activity than male mice ${ }^{36}$. We suspect that such a higher physical activity may explain this gender difference to a certain extent.

At hepatic level, activated PPAR- a enhances lipolysis, mitochondrialß-oxidation and triacylglyceride degradation ${ }^{37}$, while reducing lipogenesis through upregulation of PPAR- a with concomitant SREBP-1C downregulation ${ }^{38-40}$. In human hepatocytes, upregulation of PPAR-yrepressed expression of SREBP-2 and HMGCR which are involved in cholesterol biosynthesis ${ }^{41,42}$. In addition, activation of PPAR-yresults 
in systemic insulin sensitization through complex mechanisms involving multiple organs. In the adipose tissue, PPAR-ypromotes lipid uptake and storage. In the skeletal muscle, PPAR-yligands potentiate insulin action, and in the liver it acts to suppress gluconeogenesis, altogether leading to lower plasma glucose levels ${ }^{43-45}$. Our current data showed the good consistence with these findings.

Taken together, respective treatments in GDM dams during pregnancy with glibenclamide and metformin may be essential to increase insulin sensitivity, to lower blood lipid levels, and to improve glucose metabolism in male offspring even when they reach adulthood. Glibenclamide and metformin intervention during maternal pregnancy may have the similar intergenerational effects as do insulin intervention for male offspring. Our findings may serve the valuable reference for the GDM clinical practice.

\section{Conclusion}

Respective treatments in GDM dams during pregnancy with insulin, glibenclamide and metformin have the long-term persisted effects in male offspring, while female progenies born to untreated dams showed an autonomous inhibition of intergenerational relay of glucose and lipid dysregulation. Our current findings may shed a new light into GDM clinical practice.

\section{Abbreviations}

GDM: Gestational Diabetes Mellitus

ITT: Insulin Tolerance Test

GTT: Glucose Tolerance Test

TC: Total Cholesterol

TG: Triglycerides

HDL-c: High Density Lipoprotein-cholesterol

LDL-c: Low Density Lipoprotein-cholesterol

$A K T=P K B$. Protein Kinase $\mathrm{B}$

CPT1: Carnitine Palmitoyltransferase 1

PPAR- - : Peroxisome Proliferators-activated Receptors- $\gamma$

PEPCK: Phosphoenolpyruvate Carboxykinase

HMGCS-1: 3-Hydroxyl-3-Methylglutaryl-CoA Synthase-1 


\section{Declarations}

\section{Ethics approval and consent to participate}

Animal procedures were approved by the Animal Care and Use Committee, faculty of Science, Anhui Medical University, in accordance with the International Guiding principles for Biomedical Research Involving animals of CIOMS.

\section{Consent for publication}

All authors listed have approved the manuscript for publication

\section{Availability of data and materials}

All data in current study are available from the corresponding author on reasonable request.

\section{Competing interest}

The authors declared no conflicts of interest.

\section{Funding}

This work is supported by the National Natural Science Foundation of China (NSFC, 81570786 to K.C, 81770295 to Y.L), Wanjiang young scholar grant from Anhui Province of China (9101041203 to Z.J), Natural Scientific Research Program for Anhui Universities, and Intramural Scientific Fund for Young Scholars with Ph.D (KJ2017A827 and XJ201524 to Q.W), and The Key Research and Development Project of Anhui province of China (201904d07020003 to Y. X)

\section{Author contribution}

K.C and Y. L designed the research, provide financial support and revised/edited the manuscript; Q.W, Y.X provided partial research fund; Y. L, Z.J, and Y.J performed all experiments; J.L collected and analyzed the data; Y. L and Q.W wrote the draft of manuscript.

\section{Acknowledgement:}

We thank all the participants in the present study.

\section{References}

1. American Diabetes Association A, American PA . Diagnosis and classification of diabetes mellitus. Diab etes Care, 2013, 36(Suppl 1):S67-S74. 
2. Crowther CA, Hiller JE, Moss JR. Effect of treatment of gestational diabetes mellitus on pregnancy outcomes. N Engl J Med, 2011,60(24):2477-2486.

3. Krishnaveni GV, Veena SR, Hill JC. Intrauterine Exposure to Maternal Diabetes Is Associated With Higher Adiposity and Insulin Resistance and Clustering of Cardiovascular Risk Markers in Indian Children. 2010, 33: 402-404.

4. Portha B, Chavey A, Movassat J . Early-Life Origins of Type 2 Diabetes: Fetal Programming of the Beta-Cell Mass. Journal of Diabetes Research, 2011,1687-5214.

5. Langer O. Oral hypoglycemic agents in pregnancy: their time has come. J Matern Fetal Neonatal Med, 2002, 12(6):376-383.

6. ljas $\mathrm{H}$, Vaarasmaki M, Saarela T. A follow-up of a randomised study of metformin and insulin in gestational diabetes mellitus: growth and development of the children at the age of 18 Months. BJOG: An International Journal of Obstetrics \& Gynaecology, 2015, 122(7):994-1000.

7. Schwartz RA, Rosenn B, Aleksa K, Kore G . Glyburide Transport Across the Human Placenta. Obstetrics \& Gynecology, 2015,125(3):583-588.

8. Hod M, Kapur A, Sacks DA, Divakar H. The International Federation of Gynecology and Obstetrics (FIGO) Initiative on gestational diabetes mellitus: A pragmatic guide for diagnosis, management, and care. International Journal of Gynecology \& Obstetrics, 2015,131:S173-211.

9. NICE Guideline No.3, National Collaborating Centre for Women's and Children's Health (UK). Diabetes in pregnancy: management of diabetes and its complications from preconception to the postnatal period. https://www.nice.org.uk London, National Institute for Health and Care Excellence (UK), 2015.

10. George A, Mathews JE, Sam D. Comparison of neonatal outcomes in women with gestational diabetes with moderate hyperglycaemia on metformin or glibenclamide - A randomised controlled trial. Australian and New Zealand Journal of Obstetrics and Gynaecology, 2015, 55(1):47-52.

11. Nachum Z, Zafran N, Salim R, Yefet E. Glyburide Versus Metformin and Their Combination for the Treatment of Gestational Diabetes Mellitus: A Randomized Controlled Study. Diabetes Care, 2017, 40(3):332-337.

12. Mcllvride S, Nikolova V, Fan H, McDonald JA, Wahlström A, Bellafante E, Jansen E, Adorini L, Shapiro D, Jones XP. Marchesim Hanns-Ulrich Marschall JR, and Williamson C. Obeticholic acid ameliorates dyslipidemia but not glucose tolerance in mouse model of gestational diabetes. Am J Physiol Endocrinol Metab, 2019, 317: E399 -E410.

13. Nanobashvili K, Jack-Roberts C, Bretter R, Jones N, Axen K, Saxena A, Blain K, Jiang X. Maternal Choline and Betaine Supplementation Modifies the Placental Response to Hyperglycemia in Mice and Human Trophoblasts. Nutrients, 2018, 10: 1507-1521

14. Huang $L$, Yue P, Wu X, Chen K. Combined intervention of swimming plus metformin ameliorates the insulin resistance and impaired lipid metabolism in murine gestational diabetes mellitus. PLoS One, 2018,13(4):e0195609. 
15. Zhu H, Chen B, Cheng Y, Zhou Y, Yan YS, Luo Q, Jiang Y, Sheng JZ, Ding GL, Huang HF. Insulin therapy for gestational diabetes mellitus does not fully protect offspring from diet-induced metabolic disorders. Diabetes , 2019, 68(4): 696-708

16. Shuster DL, Risler LJ, Liang CK. Maternal-Fetal Disposition of Glyburide in Pregnant Mice Is Dependent on Gestational Age. Journal of Pharmacology and Experimental Therapeutics, 2014,350(2):425-434.

17. Jun R, Yi C, Zhen-Hua M. Intrauterine hyperglycemia exposure results in intergenerational inheritance via DNA methylation reprogramming on F1 PGCs. Epigenetics \& Chromatin, 2018, 11(1):20-28

18. Hanson RL, Imperatore G, Bennett PH, Knowler WC. Components of the "metabolic syndrome" and incidence of type 2 diabetes. Diabetes, 2002, 51(10):3120-3127.

19. Lebovitz HE. Type 2 diabetes: an overview. Clin Chem,1999,45:1339-1345

20. Xiao D, Kou H, Zhang L. Prenatal Food Restriction with Postweaning High-fat Diet Alters Glucose Metabolic Function in Adult Rat Offspring. Archives of Medical Research, 2017,48(1):35-45.

21. Danaei G, Finucane MM, Yu Y, Singh GM, Cowan MJ, Ezzati M. National, regional, and global trends in fasting plasma and diabetes prevalence since 1980: systematic analysis of health examination surveys and epidemiological studies with 370 country-years and 2.7 million participants. Lancet, 2011, 378:31-40.

22. Salpeter SR, Walsh JME, Ormiston TM, Greyber E, Buckley NS, Salpeter EE. Meta-analysis: effect of hormone-replacement therapy on components of the metabolic syndrome in postmenopausal women. Diabetes Obesity \& Metabolism, 2006, 8(5):538-554.

23. Misso M L, MurataY, Boon WC. Cellular and Molecular Characterization of the Adipose Phenotype of the Aromatase-Deficient Mouse. Endocrinology, 2003, 144(4):1474-1480.

24. Louet JF, Lemay C, Mauvais-Jarvis F. Antidiabetic actions of estrogen: Insight from human and genetic mouse models. Current atherosclerosis reports, 2004, 6(3):180-185.

25. Kim C, Kong S, Laughlin G A. Reductions in glucose among postmenopausal women who use and do not use estrogen therapy. Menopause-the Journal of the North American Menopause Society, 2013, 20(4):393-400.

26. Howard BV, Thomson CA, LaCroix AZ. The Women's Health Initiative Hormone Therapy Trials: Update and Overview of Health Outcomes During the Intervention and Post-Stopping Phases. JAMA, 2013, 310(13):1353.

27. Yan H, Yang W, Zhou F, Li X, Pan Q, Shen Z, Han G, Newell-fugate A, Gao S. Estrogen improves insulin sensitivity and suppresses gluconeogenesis via the transcription factor Foxo1. Diabetes, 2019, 68(2):291-304.

28. Yuchi Y, Cai Y, Legein B, De Groef S, Leuckx G, Van de Casteele M. Estrogen Receptor a Regulates Beta Cell Formation During Pancreas Development and Following Injury. Diabetes, 2015, 64(9),32183228.

29. Boese A C, Kim SC, Yin KJ. Sex Differences in Vascular Physiology and Pathophysiology: Estrogen and Androgen Signaling in Health and Disease. American Journal of Physiology - Heart and 
Circulatory Physiology, ajpheart.00217.2016.

30. Moretti C, Lanzolla G, Moretti M, Gnessi L, Carmina E. Androgens and Hypertension in Men and Women: a Unifying View. Current Hypertension Reports, 2017, 19(5):44.

31. Lee OD, Tillman K. An Overview of Testosterone Therapy. American Journal of Mens Health, 2016, 10(1):68-72.

32. Maseroli E, Corona G, Giagulli VA. PS-01-008 Testosterone supplementation and bodycomposition: results from a meta-analysis study. Journal of Sexual Medicine, 2016,13(5):S78-S78.

33. Kelly DM, Akhtar S, Sellers DJ. Muraleedharan V, Channer KS, Jones TH. Testosterone differentially regulates targets of lipid and glucose metabolism in liver, muscle and adipose tissues of the testicular feminised mouse. Endocrine, 2016, 54(2): 504-515.

34. Xiao D, Kou H, Zhang L. Prenatal Food Restriction with Postweaning High-fat Diet Alters Glucose Metabolic Function in Adult Rat Offspring. Archives of Medical Research, 2017, 48(1):35-45.

35. MurakamiT, ShimomuraY, Fujitsuka N, Sokabe M, Okamura K, Sakamoto S. Enlargement of glycogen store in rat liver and muscle, by fructose-diet intake and exercise training. Journal of Applied Physiology, 1997, 82(3):772-775.

36. Lightfoot JT. (2008). Sex Hormones' Regulation of Rodent Physical Activity: A Review. International Journal of Biological Sciences, 2008, 4(3):126-132.

37. Minnich A, Tian N, Byan L, Bilder G. A potent PPARalpha agonist stimulates mitochondrial fatty acid beta-oxidation in liver and skeletal muscle. Am J Physiol Endocrinol Metab. 2001, 280, E270-279.

38. Hernández-Rodas M C, Valenzuela R, Echeverría F, Rincón-Cervera MÁ, Espinosa A, Illesca P, Muñoz P, Corbari A, Romero N, Gonzalez-Mañan D, Videla LA. Supplementation with Docosahexaenoic Acid and Extra Virgin Olive Oil Prevents Liver Steatosis Induced by a High-Fat Diet in Mice through PPAR-a and Nrf2 Upregulation with Concomitant SREBP-1c and NF-kB Downregulation. Molecular Nutrition \& Food Research, 2017, 61(12), doi: 10.1002/mnfr.201700479.

39. RenT, Zhu J, Zhu L, Cheng M. The Combination of Blueberry Juice and Probiotics Ameliorate NonAlcoholic Steatohepatitis (NASH) by Affecting SREBP-1c/PNPLA-3 Pathway via PPAR-a. Nutrients, 2017, 9(3): pii: E198.

40. Lee JH, Kang HS, Park HY, MoonYA, Kang YN, Oh BC, Song DK, Bae JH, Im SS. PPARa-dependent Insig2a overexpression inhibits SREBP-1c processing during fasting. Scientific Reports , 2017, 7(1):9958.

41. HanT, LvY, Wang S, Hu T, Hong H, Fu Z. PPARY overexpression regulates cholesterol metabolism in human L02 hepatocytes. J Pharmacol Sci, 2019, 139:1-8.

42. Klopotek A., Hirche F, Eder K. PPARy Ligand Troglitazone Lowers Cholesterol Synthesis in HepG2 and Caco-2 Cells via a Reduced Concentration of Nuclear SREBP-2. Experimental Biology and Medicine, 2006, 231(8):1365-1372.

43. Diaz-Delfin J, Morales M, Caelles C. Hypoglycemic Action of Thiazolidinediones/Peroxisome Proliferator-Activated Receptor by Inhibition of the c-Jun NH2-Terminal Kinase Pathway. Diabetes, 2007, 56(7):1865-1871. 
44. Hevener AL, Olefsky JM, Reichart D, Nguyen MT, Bandyopadyhay G, Leung HY, Watt MJ, Benner C, Febbraio MA, Nguyen AK, Folian B, Subramaniam S, Gonzalez FJ, Glass CK, Ricote M. Macrophage PPARy is required for normal skeletal muscle and hepatic insulin sensitivity and full antidiabetic effects of thiazolidinediones. Journal of Clinical Investigation, 2007, 117(6):1658-1669.

45. Odegaard JI, Ricardo-Gonzalez RR, Goforth MH, Morel CR, Subramanian V, Mukundan L, Red Eagle A, Vats D, Brombacher F, Ferrante AW, Chawla A. Macrophage-specific PPARgamma controls alternative activation and improves insulin resistance. Nature, 2007, 447(7148):1116-1120.

\section{Figures}



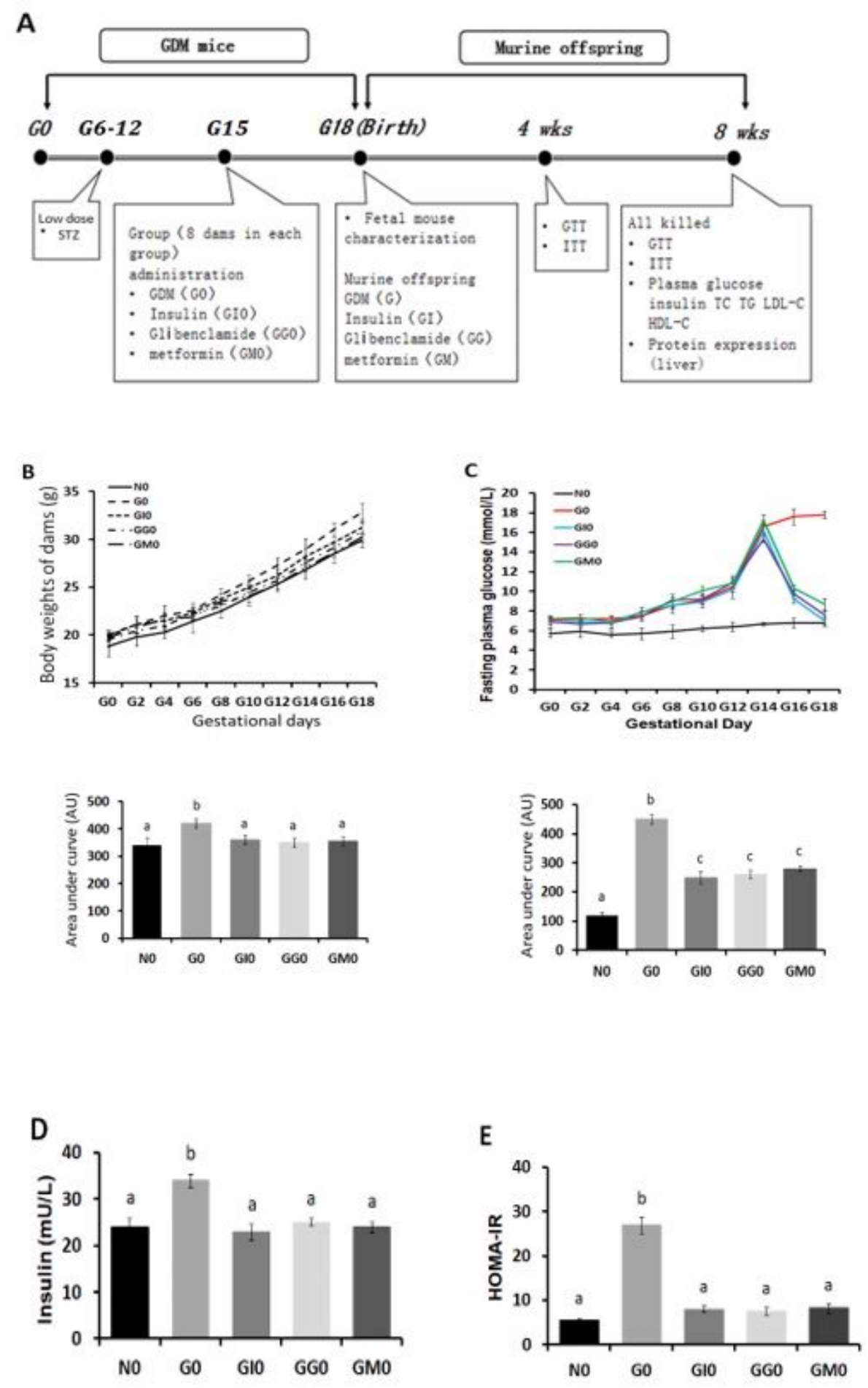

\section{Figure 1}

Respective interventions of insulin, glibenclamide and metformin during pregnancy corrected the plasma glucose and insulin resistance in GDM dams A, The showed is the whole experimental scheme starting from the establishment of maternal GDM model to the adulthood of offspring. B, The growth curves of body weights of GDM and control maternal mice during pregnancy. C, The dramatic elevation of fasting plasma glucose level was induced by GDM for dams during pregnancy, which could be substantially 
improved by respective treatments of insulin, glibenclamide and metformin. D, GDM remarkably raised the plasma insulin level in maternal mice, while the respective treatments normalized the level. E, GDM sharply enhanced the HOMA-IR level in untreated maternal mice, the respective treatments, however, greatly improved the level. The values represent the mean $\pm S E M, n=8-10$ dams/group. The columns or curves with different letters $(a, b, c)$ are significantly different, $P<0.05$ (ANOVA, SNK).
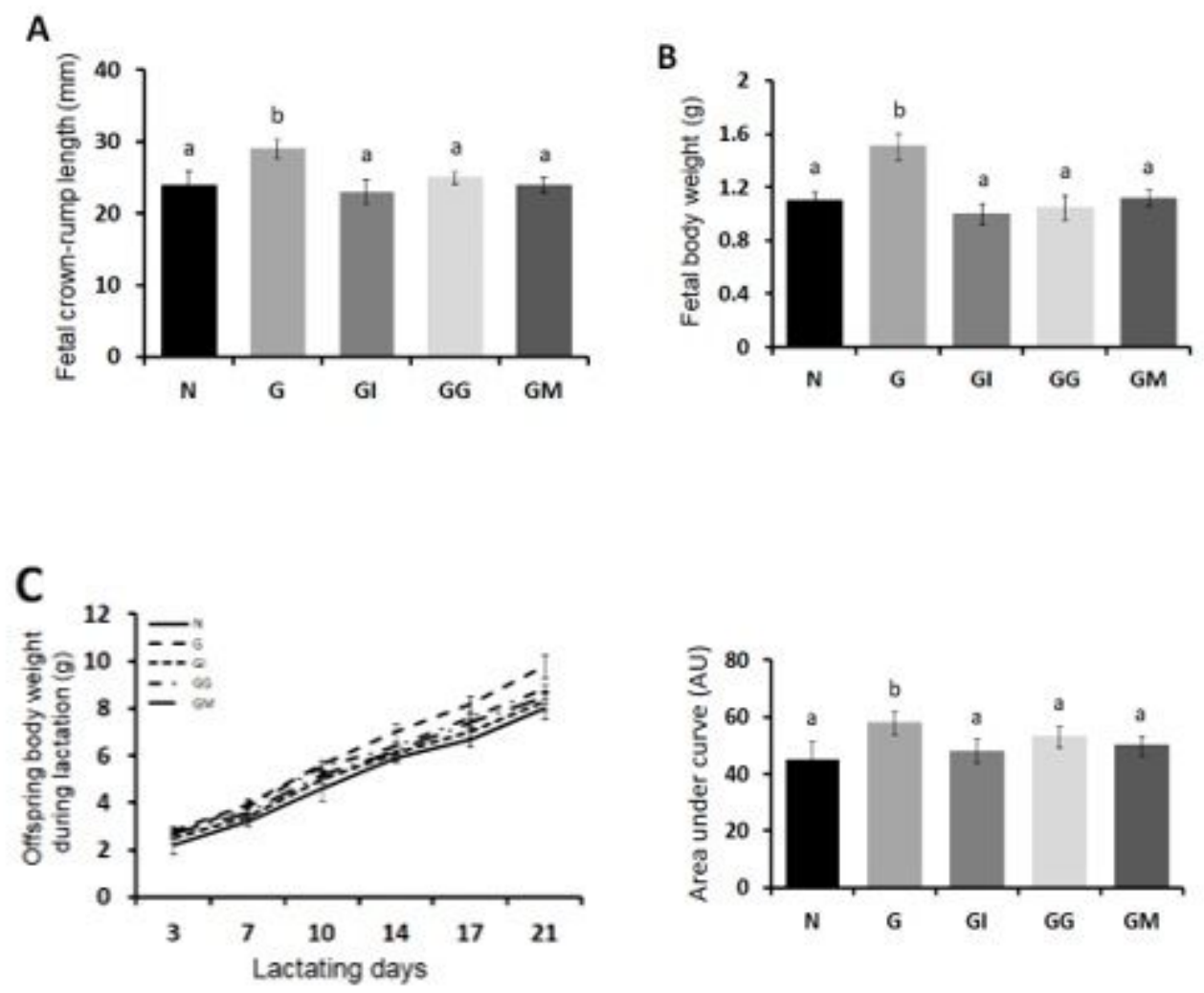

\section{Figure 2}

Insulin, glibenclamide and metformin respectively normalized fetal body weights and GDM-induced overgrowth in progenies during lactation A, Fetal crown-rump length was significantly higher in untreated $G$ group than in control group that could be normalized by three drug interventions. B, GDM induced greatly higher fetal body weights than that in healthy control, which could be corrected by three drug therapies. C, The growth rates of body weight in offspring born to untreated dams were significantly higher than those in control and drug-treated groups during lactation. The values represent the mean \pm SEM, $n=8-10$ pups/group. The columns or curves with different letters $(a, b)$ are significantly different, $P<0.05$ (ANOVA, SNK). 
A

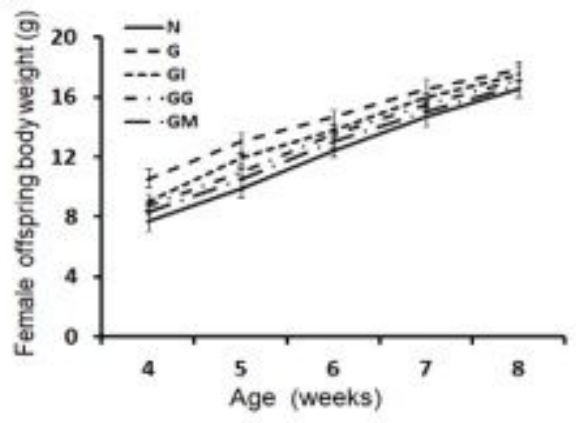

C
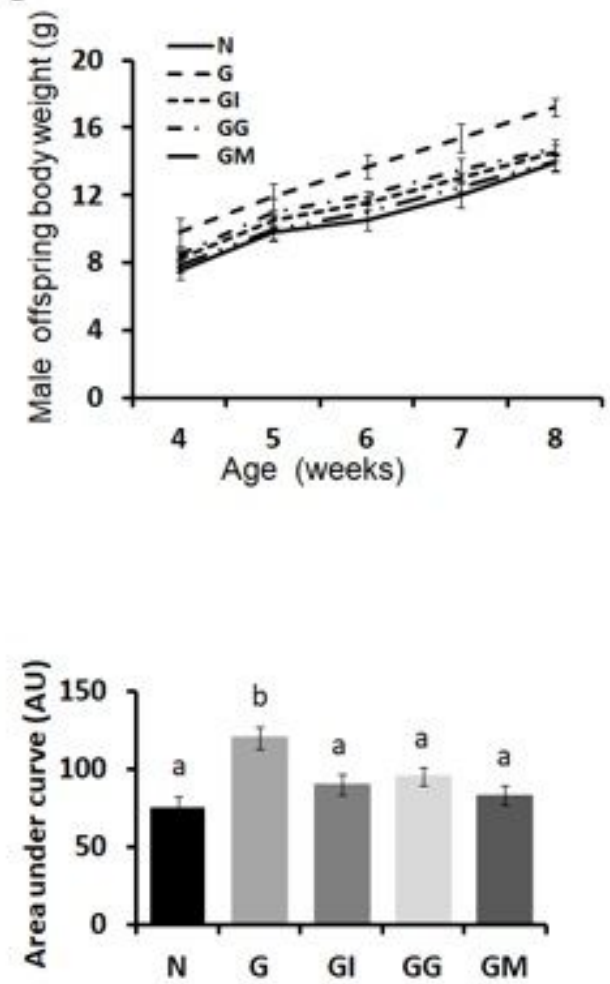

B

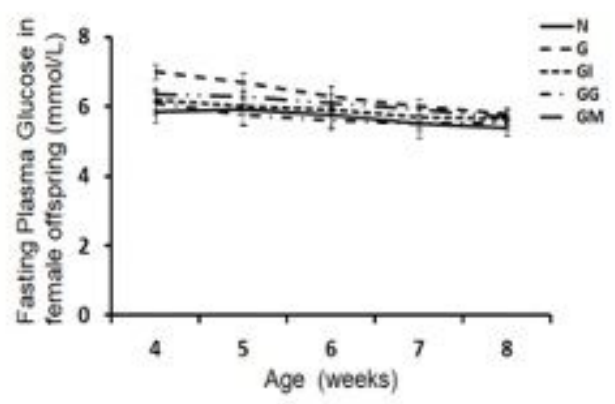

D
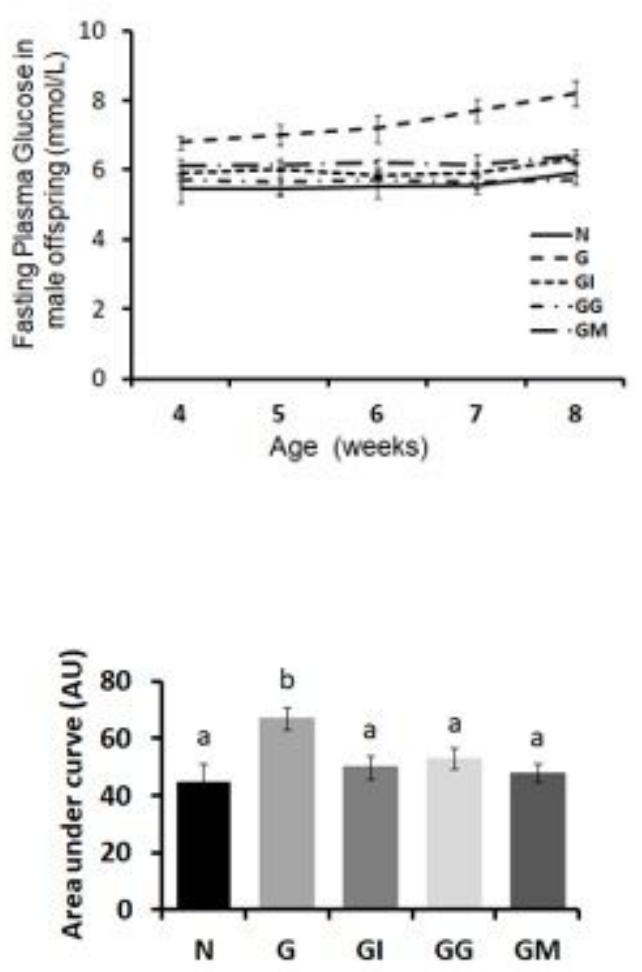

\section{Figure 3}

Respective therapies of three drugs during maternal pregnancy normalized the fasting plasma glucose levels in male adult offspring. A, Growth curves of female offspring in different groups from 4 to 8 weeks of age showed an autonomous correction of overgrowth caused by untreated GDM in dams. B, Fasting plasma glucose changes in female offspring in different groups from 4 to 8 weeks of age indicated an autonomous improvement of hyperglycemia induced by untreated GDM in dams. C, Growth curves of male offspring in different groups from 4 to 8 weeks of age exhibited significant inhibition of overgrowth by the respective three drug treatments. D, Fasting plasma glucose changes in male offspring in different groups from 4 to 8 weeks of age suggested the dramatic suppression of hyperglycemia by three drug 
therapies. The values represent the mean \pm SEM, $n=8-10$ /group. The curves with different letters $(a, b)$ are significantly different, $\mathrm{P}<0.05$ (ANOVA, SNK).
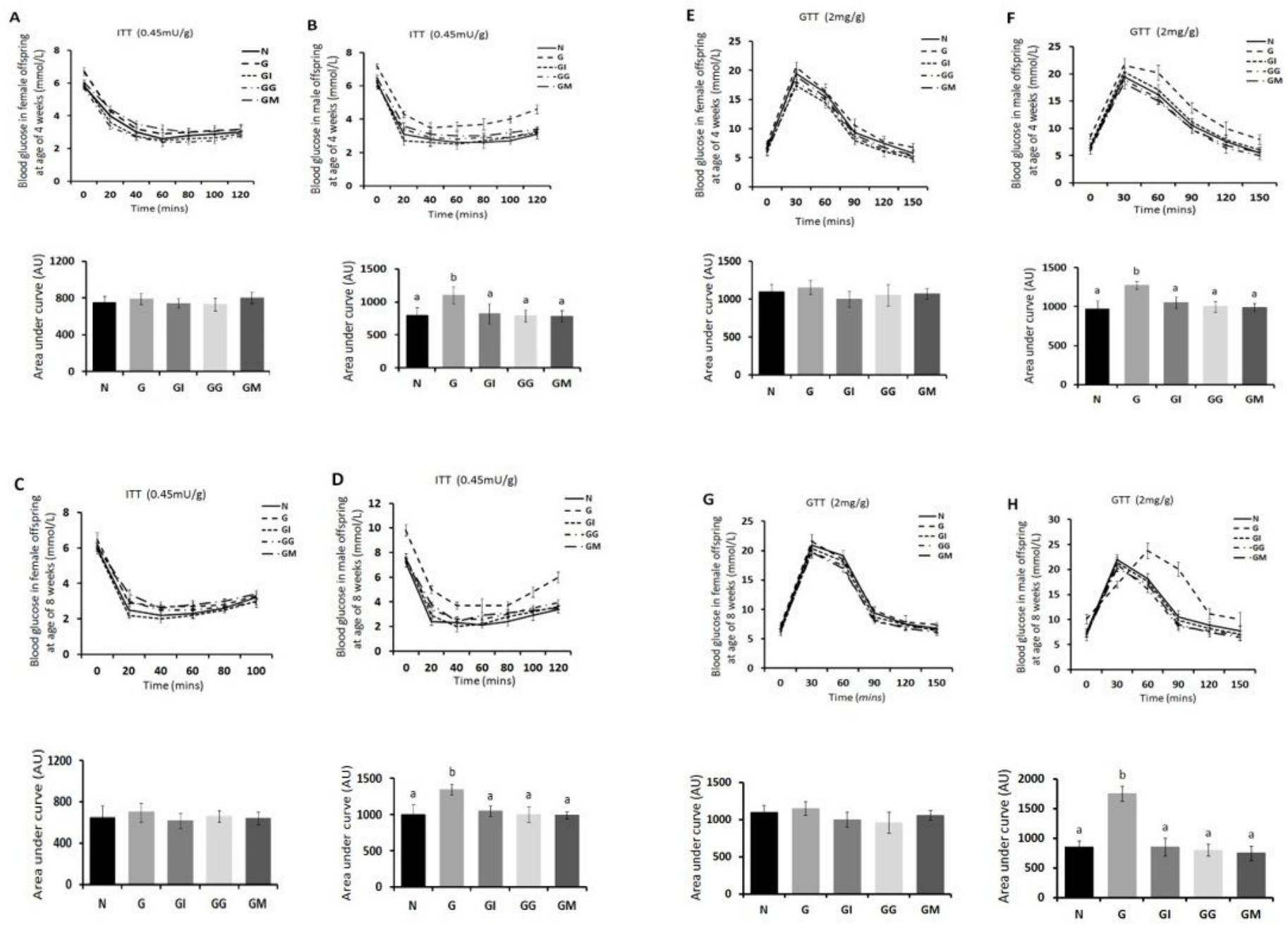

\section{Figure 4}

Normalization of glucose tolerance and insulin sensitivity by the respective interventions of three drugs in dams only persisted in male offspring. A, Insulin tolerance test in female offspring in different groups at 4 weeks of age. The mice were fasted $6 \mathrm{~h}$ on 4 th week and injected with insulin at $0.45 \mathrm{mU} / \mathrm{g}$ body weight, and glucose levels were determined at 0,20, 40,60,80 and 120 min after injection. Quantified results are expressed as the area under the curve. $B$, Insulin tolerance tests were conducted in male offspring in different groups at 4 weeks of age. C, Insulin tolerance tests were performed in female offspring in different groups on 8th week of age. D, Insulin tolerance tests were done in male offspring in different groups on 8th week of age. E, Intraperitoneal glucose tolerance tests were executed in female offspring in different groups on 4th week of age. The mice were fasted $6 \mathrm{~h}$ on 4th week and injected with glucose at $2 \mathrm{mg} / \mathrm{g}$ body weight, and plasma glucose were determined at 0,30,60, 90,120 and 150min after injection. Quantified results are expressed as the area under the curve. F, The showed are the intraperitoneal glucose tolerance tests (GTT, $2 \mathrm{mg} / \mathrm{g}$ ) in male offspring in different groups on 4th week of age. G, 
Intraperitoneal glucose tolerance tests (GTT, $2 \mathrm{mg} / \mathrm{g}$ ) were conducted in female offspring in different groups on 8th week of age. $H$, The exhibited are the intraperitoneal glucose tolerance tests (GTT, $2 \mathrm{mg} / \mathrm{g}$ ) in male offspring in different groups on 8th week of age. The same procedure as A was taken for B, C, D, respectively; and the same procedure as $E$ was taken for $F, G, H$, respectively. The values represent the mean $\pm S E M, n=8-10 /$ group. The columns or curves with different letters $(a, b)$ are significantly different, $\mathrm{P}<0.05$ (ANOVA, SNK).
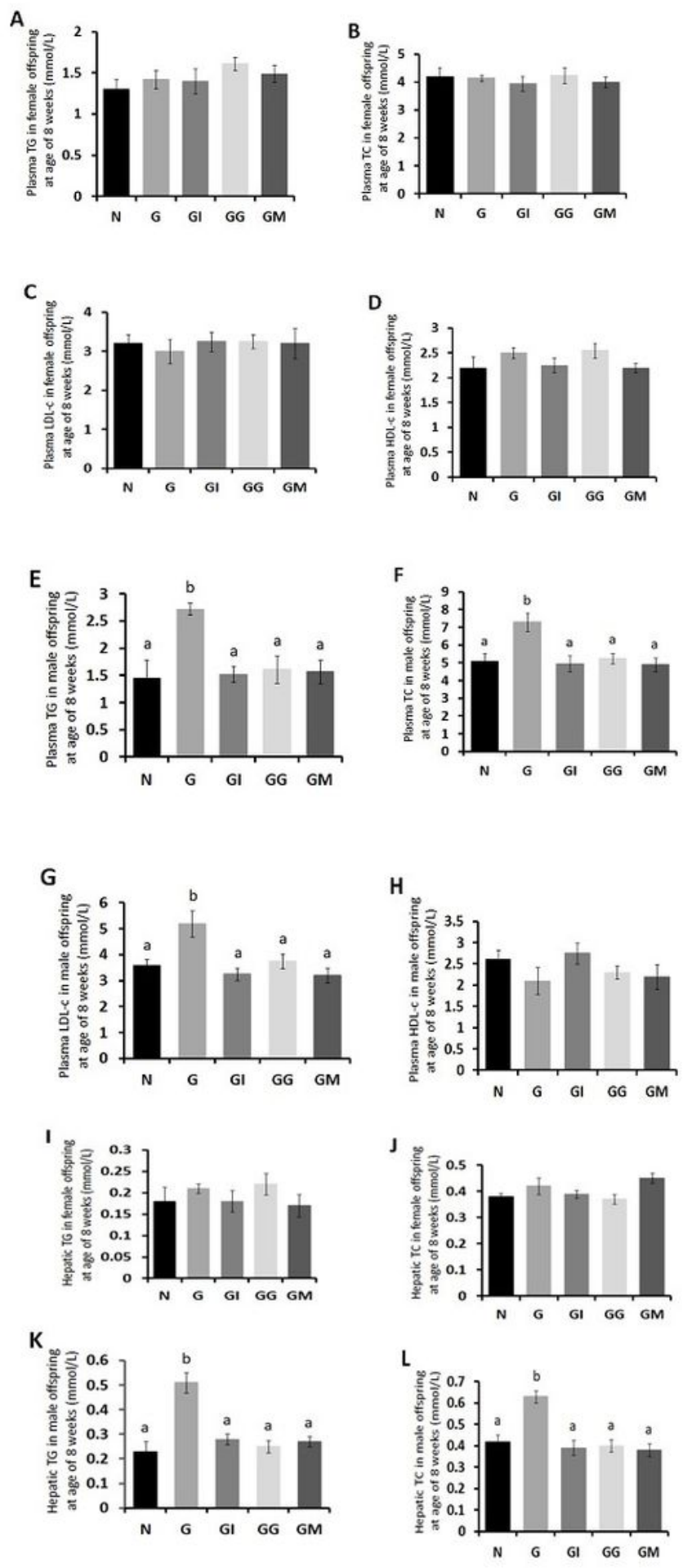

Figure 5 
Normalization of blood and hepatic lipid levels by respective interventions of three drugs during maternal pregnancy only persisted in male offspring at adulthood. A, The demonstrated are plasma TG levels in female offspring in different groups on 8th week. B, The showed are plasma TC levels in female offspring in different groups on 8th week. C, Plasma LDL-C levels were detected in female offspring in different groups on 8th week. D, Plasma HDL-C levels were assayed in female offspring in different groups on 8th week. E, The exhibited are plasma TG levels in male offspring in different groups on 8th week. F, Plasma TC levels were analyzed in male offspring in different groups on 8th week. $G$, The showed are plasma LDL-C levels in male offspring in different groups on 8th week. $H$, The detected are plasma HDL-C levels in male offspring in different groups on 8th week. I, Hepatic TG levels were assayed in female offspring at 8 weeks of age. J, Hepatic TC levels were analyzed in female offspring at 8 weeks of age. K, Hepatic TG levels were detected in male offspring at 8 weeks of age. L, The showed are hepatic TC levels in male offspring at 8 weeks of age. The values represent the mean $\pm S E M, n=8-10 /$ group. The columns with different letters $(a, b)$ are significantly different, $P<0.05$ (ANOVA, SNK).
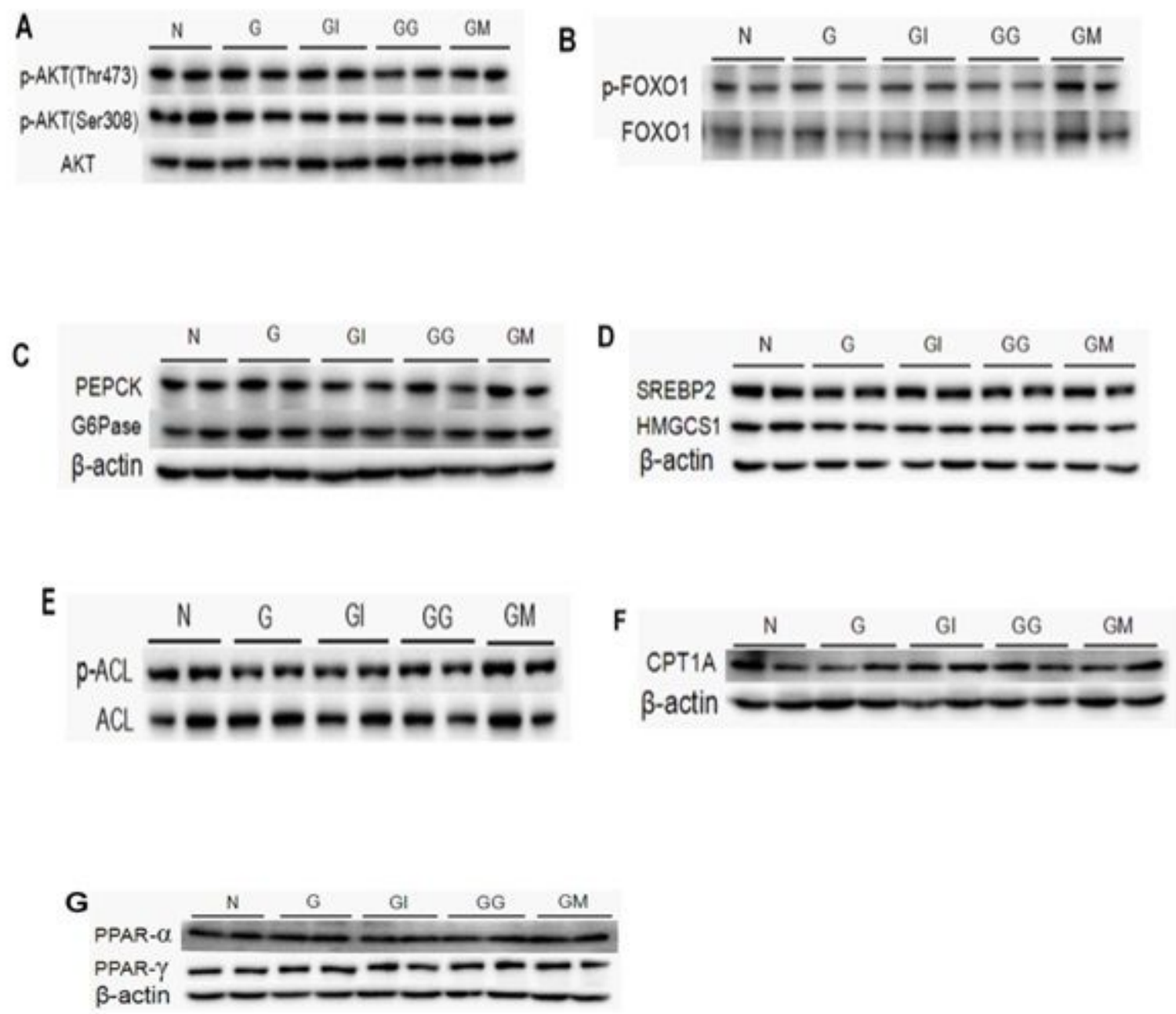

\section{Figure 6}

The female offspring born to untreated GDM maternal mice displayed autonomous reinstatement of insulin sensitivity, glucose and lipid regulation when reached their adulthood. A, Levels of phosphorylated 
AKT (p-AKT S473, p-AKT T308) were restored in the livers of female offspring born to the untreated GDM dams at 8 weeks of age. B, Levels of phosphorylated FOXO1 were rehabilitated in the livers from female offspring born to the untreated GDM dams at 8 weeks of age. C, Protein expressions of PEPCK and G6Pase were retrieved in the livers of female offspring born to the untreated GDM dams at 8 weeks of age. D, Protein expressions of SREBP2 and HMGCS1 were normalized in the livers of female offspring born to the untreated GDM dams at 8 weeks of age. E, Levels of phosphorylated ACL were recovered in the livers of female offspring born to the untreated GDM dams at 8 weeks of age. F, Protein expression of CPT1A was restored in the livers of female offspring born to the untreated GDM dams at 8 weeks of age. $G$, Levels of hepatic PPAR- $\alpha$ and PPAR- $\gamma$ were reinstated in female offspring born to the untreated GDM dams at 8 weeks of age. All data in this figure are representatives of three independent experiments.
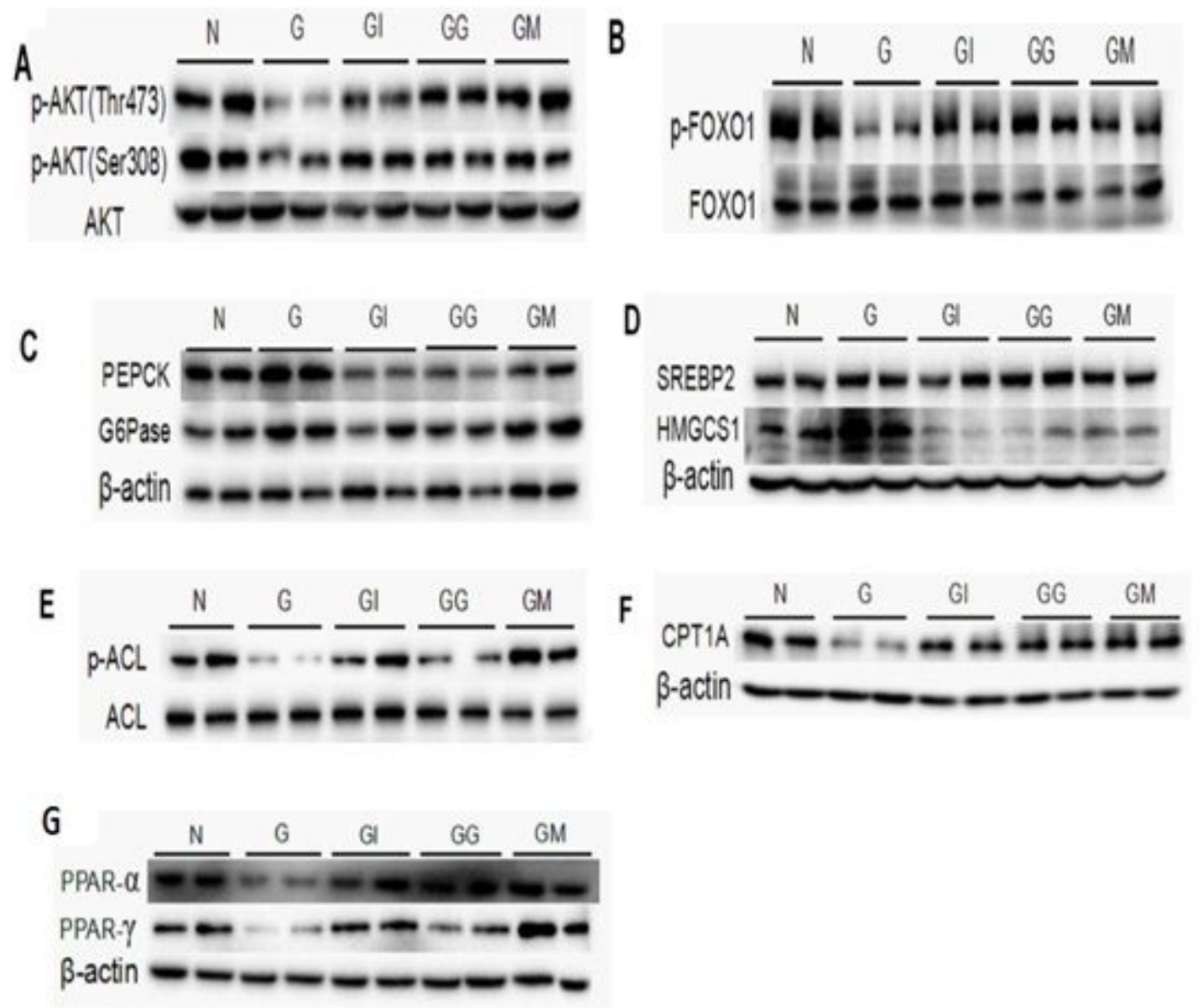

\section{Figure 7}

The normalization of hepatic insulin signaling, gluconeogenesis and de novo lipogenesis by the drug interventions during maternal pregnancy still persisted in male offspring at adulthood. $A$, The decreased levels of phosphorylated AKT (p-AKT S473, p-AKT T308) caused by maternal GDM were drastically improved in the livers of drug-treated male offspring at 8 weeks of age. B, The inhibited levels of phosphorylated FOXO1 caused by maternal GDM were dramatically enhanced in the livers of drug-treated male offspring at 8 weeks of age. C, The elevated protein expressions of PEPCK and G6Pase in G group were substantially suppressed in the livers of male offspring treated with the drugs at 8 weeks of age. D, 
The boosted protein expression of HMGCS1 not SREBP2 caused by maternal GDM was sharply improved in the livers of drug-treated male offspring at 8 weeks of age. E, The enhanced levels of phosphorylated ACL caused by maternal GDM were markedly improved in the livers of drug-treated male offspring at 8 weeks of age. F, The suppressed protein expression of CPT1A caused by maternal GDM was substantially reinstated in the livers of drug-treated male offspring at 8 weeks of age. All data in this figure are representatives of three independent experiments.

\section{Supplementary Files}

This is a list of supplementary files associated with this preprint. Click to download.

- SIFigure.jpg 